Software Engineering

\footnotetext{
Software ENgineering

SEN Synthesis of Reo Circuits For Implementation Of

Component-Connector Automata Specifications

F. Arbab, C. Baier, F.S. de Boer, J.J.M.M. Rutten, M. Sirjani
}

RePORt SEN-R0412 JuLY 2004 
CWI is the National Research Institute for Mathematics and Computer Science. It is sponsored by the Netherlands Organization for Scientific Research (NWO).

$\mathrm{CWI}$ is a founding member of ERCIM, the European Research Consortium for Informatics and Mathematics.

CWI's research has a theme-oriented structure and is grouped into four clusters. Listed below are the names of the clusters and in parentheses their acronyms.

Probability, Networks and Algorithms (PNA)

\section{Software Engineering (SEN)}

Modelling, Analysis and Simulation (MAS)

Information Systems (INS)

Copyright ( 2004 , Stichting Centrum voor Wiskunde en Informatica

P.O. Box 94079, 1090 GB Amsterdam (NL)

Kruislaan 413, 1098 SJ Amsterdam (NL)

Telephone +31 205929333

Telefax +31205924199

\section{ISSN 1386-369X}




\title{
Synthesis of Reo Circuits For Implementation Of Component-Connector Automata Specifications
}

\begin{abstract}
Composition of a concurrent system out of components involves coordination of their mutual interactions. In component-based construction, this coordination becomes the responsibility of the glue-code language and its underlying run-time middle-ware. Reo offers an expressive gluelanguage for construction of coordinating component connectors out of primitive channels. In this paper we consider the problem of synthesizing Reo coordination code from a specification of a behavior as a relation on scheduled-data streams. The specification is given as a constraint automaton that describes the desired input/output behavior at the ports of the components. We present an algorithm that generates Reo code from a given constraint automaton.
\end{abstract}

1998 ACM Computing Classification System: C.2.4, D.1.3, D.2.4, D.2.6, D.2.11, D.2.13, D.3.2, D.3.3, F.1.2, F.3.1, F.3.2, F.3.3

Keywords and Phrases: Coordination, Composition, Reo, Constraint Automata, Timed Data Streams, Connector Gluecode Synthesis 


\title{
Synthesis of Reo Circuits For Implementation Of Component-Connector Automata Specifications
}

\author{
Farhad Arbab $^{a, c}$, Christel Baier $^{b}$, Frank de Boer $^{a, c}$, Jan Rutten $^{a, d}$, Marjan Sirjani ${ }^{e}$ \\ ${ }^{a}$ CWI, Amsterdam, The Netherlands \\ ${ }^{b}$ Universität Bonn, Institut für Informatik I, Germany \\ ${ }^{c}$ Universiteit Leiden, The Netherlands \\ ${ }^{d}$ Vrij Universiteit, Amsterdam, The Netherlands \\ ${ }^{e}$ Sharif University of Technology, Tehran, Iran \\ \{farhad / janr / frb / msirjani\}@cwi.nl \\ baier@cs.uni-bonn.de
}

\begin{abstract}
Composition of a concurrent system out of components involves coordination of their mutual interactions. In component-based construction, this coordination becomes the responsibility of the glue-code language and its underlying run-time middle-ware. Reo offers an expressive glue-language for construction of coordinating component connectors out of primitive channels. In this paper we consider the problem of synthesizing Reo coordination code from a specification of a behavior as a relation on scheduled-data streams. The specification is given as a constraint automaton that describes the desired input/output behavior at the ports of the components. We present an algorithm that generates Reo code from a given constraint automaton.
\end{abstract}

Keywords and phrases: Coordination, Composition, Reo, Constraint Automata, Timed Data Streams, Connector Glue-code Synthesis

1998 ACM Computing Classification: C.2.4, D.1.3, D.2.4, D.2.6, D.2.11, D.2.13, D.3.2, D.3.3, F.1.2, F.3.1, F.3.2, F.3.3

\section{Introduction}

Composing components into a concurrent system involves coordination of their mutual interactions. The internals of black-box components cannot be modified to implement such coordinated interactions. Coordination, therefore, becomes the responsibility of the "glue-code" that inter-connects the constituent components of a composite system, and of its underlying run-time middle-ware. Reo [3] offers a powerful glue language for implementation of coordinating component connectors that resemble electronic circuits and are based on a calculus of mobile channels. Reo is being used, for instance, in the context of the Cybernetic Incident Management project [11] for composition of web services which constitute the black-box components of dynamically configured distributed applications [13]. In this paper, we address the synthesis problem of component connectors with Reo as our target implementation language. The input for this problem is a specification of a coordination protocol and its output is a Reo connector circuit that implements this protocol.

Synthesis problems address the question of the (algorithmic) generation of an implementation from a given specification and have a long tradition in computer science. In the context of switching circuits, the synthesis problem was first raised by Church [10] and is nowadays well-understood; see, e.g., [9, 23, 19, 17]. For temporal logical specifications, several synthesis algorithms have been suggested that rely on the close relationship between the synthesis and satisfiability problem $[15,20,7,22,6]$ or on a game-theoretic view where a system must be designed to meet a specification, no matter how an opponent (the environment) behaves [22, 1, 14, 24, 27, 26, 16]. The output of these synthesis algorithms are some kind of automata or state-transition graphs. Our goal is a step further toward 
an implementation by generating Reo code from a given automaton specification. Thus, our contribution is more in the spirit of gate-level hardware synthesis from given automata specifications.

More precisely, our starting point is a specification of a component connector as a relation over timed data streams $[8,5]$, represented by a constraint automaton [4]. Constraint automata are variants of labeled transition systems that operationally describe the maximally parallel data-flow activity through the nodes in a Reo circuit. In [4], constraint automata are used to provide an operational semantics for coordination mechanisms formalized by composition of Reo connector graphs. In a constraint automaton, the states of the automaton represent the possible configurations (e.g., the contents of the FIFO-channels of the Reo-connector); transitions going out of a state represent data-flow at that state and its effect on the configuration.

In this paper we are not primarily concerned with the derivation of (constraint) automata representations from higherlevel behavior specifications, such as in temporal logic or relations on timed data streams. Similar derivations, for instance, in the field of digital circuit design, are well-known. The main contribution of this paper is an algorithm that takes as input a constraint automaton $\mathcal{A}$ and produces a Reo connector graph that implements the relation on timed data streams specified by $\mathcal{A}$. This is tantamount to compiling an automaton down to actual concurrent executable code for a distributed implementation of the coordination behavior specified by that automaton. To establish this result, we first transform $\mathcal{A}$ into an equivalent scheduled-data expression which is a slight variant of an ordinary $\omega$ regular expression. We then construct circuits for the atomic expressions and composition operators on Reo circuits that capture the semantics of concatenation, union, and infinity-closures. Under certain (weak) conditions, the size of the resulting circuit is linear in the length of the given expression.

Superficially, compiling constraint automata specifications to Reo circuits seems simple. By analogy, derivation of digital circuits from Mealy automata specifications are well understood. However, constraint automata (and Reo circuits) can exhibit far more complex behavior than digital circuits, including combinations of synchrony and asynchrony, and relational, as well as simple (input/output) functional, interdependencies. In the light of this fact, it is far from obvious if synthesis of Reo circuits from constraint automata is possible at all, and if so, whether it can be done efficiently.

The rest of this paper is organized as follows. Section 2 summarizes the main features of Reo. Section 3 recalls the definition of constraint automata and their accepted TDS-languages. In Section 4, we show the equivalence of scheduled-data expressions and constraint automata. The construction of a Reo circuit from a given expression is explained in Section 5. Section 6 concludes the paper.

\section{A Reo primer}

Reo [3] is a channel-based exogenous coordination model wherein complex coordinators, called connectors, are compositionally built out of simpler ones. The simplest connectors in Reo are a set of channels with well-defined behavior supplied by users. Components can instantiate, compose, connect to, and perform I/O operations through connectors. Here, as in [5, 4], we do not consider the dynamic creation, composition, and reconfiguration of connectors by components. We restrict our attention to connectors that have a static graphical representation as a Reo circuit which coordinates the data-flow through the channels connecting the input/output ports of components.

Reo's notion of channel is far more general than its common interpretation and allows for any primitive communication medium with exactly two ends. The channel ends are classified as source ends through which data enters and sink ends through which data leaves a channel. Although Reo allows for an open-ended set of channel-types with user-defined semantics, for our purposes in this paper, we restrict ourselves to the channel-types shown in Fig. 1.

The simplest form of an asynchronous channel is a FIFO channel with one buffer cell (called a 1-bounded FIFO channel or simply a FIFO1 channel). We graphically represent a FIFO1 channel by a small box in the middle of an arrow. In the example in Fig. 1, the left channel-end is a source, and the right end is a sink. The buffer is assumed to be initially empty if no data item is shown in the box (this is the case in Fig. 1). The graphical representation of a FIFO1-channel whose buffer initially contains a data element $d$ shows $d$ inside the box. FIFO channels with two or more buffer cells can be produced by composing several FIFO1 channels, as for instance, explained in [5, 4].

A synchronous channel (depicted as a simple solid arrow) has a source and a sink end, and no buffer. It accepts a data item through its source end iff it can simultaneously dispense it through its sink. A lossy synchronous channel 


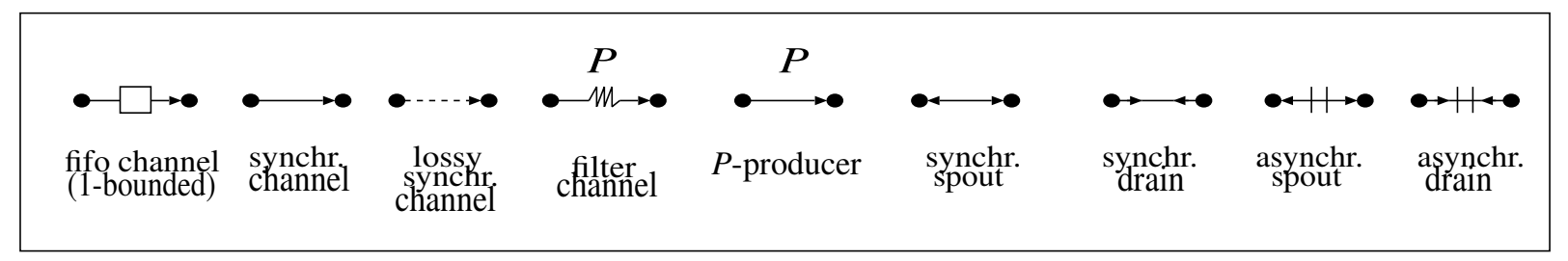

Figure 1: Basic channel-types in Reo

(depicted as a dashed arrow) is similar to a synchronous channel, except that it always accepts all data items through its source end. If it is possible for it to simultaneously dispense the data item through its sink (e.g., there is a take operation pending on its sink) the channel transfers the data item; otherwise the data item is lost. For a synchronous filter channel, its "pattern" $P$ (for our purposes here, formalized as a set $P \subseteq$ Data) specifies the type of data items that can be transmitted through the channel. Any value $d \in P$ is accepted through its source end iff its sink end can simultaneously dispense $d$; all data items $d \notin P$ are always accepted through the source end but are immediately lost. The $P$-producer is a variant of a synchronous channel whose source end accepts any data item $d \in D a t a$, but the value dispensed through its sink end is always a data element $d \in P$.

More exotic channels permitted in Reo are (a)synchronous drains that have two source ends. Because a drain has no sink end, no data value can ever be obtained from these channels. Thus, a synchronous drain accepts a data item through one of its ends iff a data item is also available for it to simultaneously accept through its other end as well. All data accepted by this channel are lost. An asynchronous drain accepts and loses data items through its two source ends, but never simultaneously. Synchronous and asynchronous spouts are duals of their corresponding drain channel types, as they have two sink ends.

A complex connector has a graphical representation, called a Reo circuit, which can be produced by applying certain composition operators to channels. In our setting, where we do not consider dynamic aspects of the Reo language, a Reo-circuit is a finite graph where the nodes are labeled with pair-wise disjoint, non-empty sets of channel ends, and where the edges represent their connecting channels. The set of channel ends coincident on a node $A$ is disjointly partitioned into the sets $\operatorname{Src}(A)$ and $\operatorname{Snk}(A)$, denoting the sets of source and sink channel ends that coincide on $A$, respectively. A node is called a source node if $\operatorname{Src}(A) \neq \emptyset \wedge \operatorname{Snk}(A)=\emptyset$. Analogously, $A$ is called a sink node if $\operatorname{Src}(A)=\emptyset \wedge \operatorname{Snk}(A) \neq \emptyset$. Node $A$ is called a mixed node if $\operatorname{Src}(A) \neq \emptyset \wedge \operatorname{Snk}(A) \neq \emptyset$. In this paper, it suffices to assume that all mixed nodes are hidden. In other words, we abstract away from their names and formalize the behavior of a Reo circuit by means of the data-flow at its sink and source nodes. Intuitively, source nodes of a circuit are analogous to the input ports, and sink nodes to the output ports of a component, while mixed nodes are its hidden internal details. Components cannot connect to, read from, or write to mixed nodes. Instead, data-flow through mixed nodes is totally specified by the circuits they belong to.

A component can write data items to a source node of a Reo circuit that it is connected to. A write operation succeeds only if all (source) channel ends coincident on the node accept the data item, in which case the data item is transparently written to every source end coincident on the node. A source node, thus, acts as a replicator. A component can obtain data items from a sink node of a Reo circuit that it is connected to through input operations. ${ }^{1}$ A take operation succeeds only if at least one of the (sink) channel ends coincident on the node offers a suitable data item; if more than one coincident channel end offers suitable data items, one is selected nondeterministically. A sink node, thus, acts as a nondeterministic merger. A mixed node is a self-contained "pumping station" that combines the behavior of a sink node (merger) and a source node (replicator) in an atomic iteration of an endless loop: in every iteration a mixed node nondeterministically selects and takes a suitable data item offered by one of its coincident sink channel ends and replicates it into all of its coincident source channel ends. A data item is suitable for selection in an iteration only if it can be accepted by all source channel ends that coincide on the mixed node.

Example 2.0.1 (Exclusive router and shift-lossy FIFO1 channel) Fig. 2 a. shows an implementation of an exclusive router built by composing five synchronous channels, two lossy synchronous channels and a synchronous drain. The intuitive behavior of this circuit is that through its source node A, it obtains a data item d from its environment and delivers $d$ to one of its sink nodes $B$ or $C$. If both $B$ and $C$ are willing to accept $d$ then the exclusive router

\footnotetext{
${ }^{1}$ We consider only the destructive take operation here which, e.g., on a FIFO channel, reads and removes the first data item in its buffer.
} 


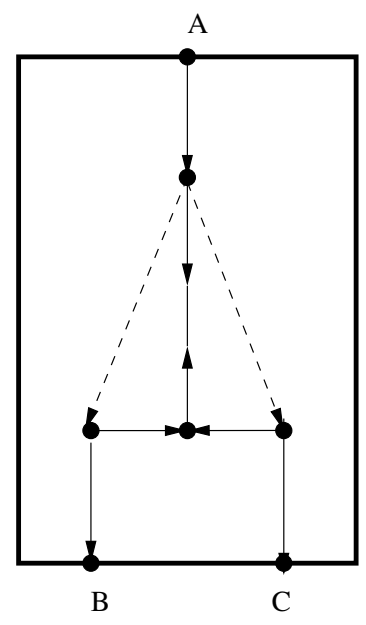

$\mathbf{a}$

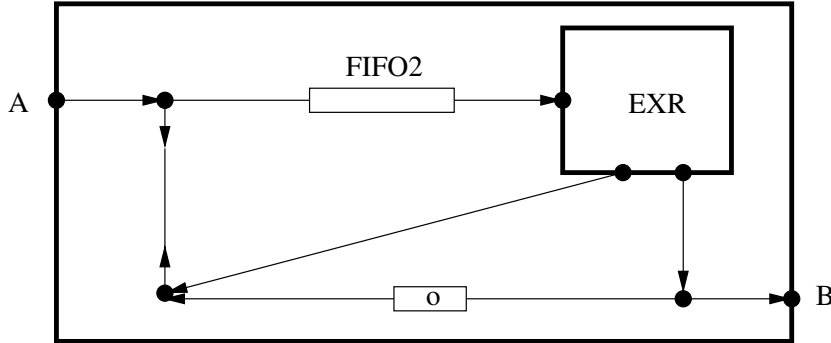

b

Figure 2: Exclusive router and shift-lossy FIFO1 channel

nondeterministically decides to deliver $d$ to either $B$ or $C$. The circuit in Fig. 2.b shows an implementation of a shiftlossy FIFO1 channel with source node A and sink node B. This implementation uses four synchronous channels, a synchronous drain, a FIFO1 channel whose buffer initially contains a token data item, o, an empty FIFO2 channel, and an instance of the exclusive router of Fig. 2.a shown as the box labeled EXR. A shift-lossy FIFO1 channel behaves the same as a FIFOI channel, except that writing to its source end is never blocked. If at the time of a write operation its buffer is full, the stored data item in the buffer is lost and the new data item replaces it in the buffer. The observable behavior of each of these Reo circuits is represented by a constraint automaton in Fig. 3. Derivation of these constraint automata as compositions of the constraint automata representing the behavior of the individual primitives used in their respective Reo circuits appears in [4].

In spite of its simplicity, the semantics of Reo is indeed very rich, yielding a surprisingly expressive language [3]. For instance, the relational (as opposed to functional) dependencies that result in "propagation of synchrony" as well as the way in which the local behavior of, e.g., lossy synchronous channels imposes non-local constraints on a circuit, are already evident in the exclusive router of Fig. 2.a. (We use this exclusive router later in this paper in our synthesis of Reo circuits.) Examples of Reo circuits with more interesting behavior can be found elsewhere [2], and the reader is encouraged to see [25] and [5] for the simple, rich, and expressive formal semantics of Reo.

In the remainder of the paper, we discuss the synthesis problem of Reo circuits where the input specification of the desired coordination is given as a constraint automaton, as defined in the next section.

\section{Constraint automata}

Constraint automata can serve as an operational model for Reo circuits [4]. The states of an automaton represent the configurations of its corresponding circuit (e.g., the contents of the FIFO channels), while the transitions encode its maximally-parallel stepwise behavior. The transitions are labeled with the maximal sets of nodes on which data-flow occurs simultaneously, and a data constraint (i.e., boolean condition for the observed data values).

We start with a simple example for a constraint automaton that models a component with input port $A$ and two output ports $B$ and $C$ which is modelled by a Reo circuit as shown in the left of the picture below. 

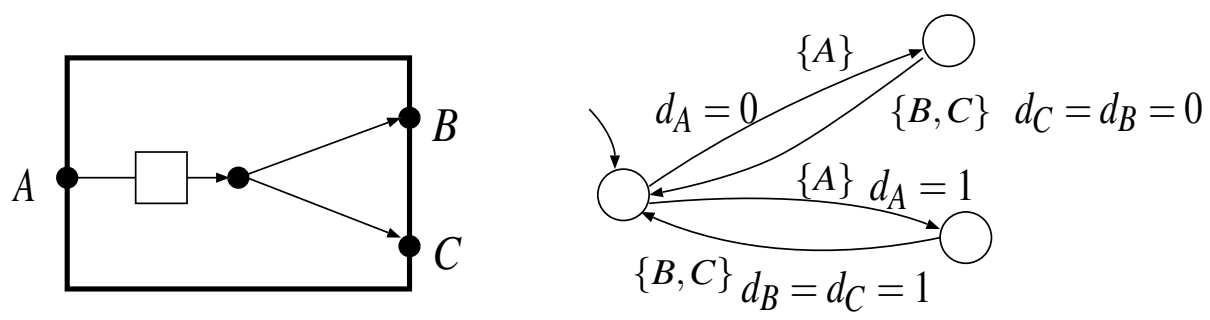

The picture on the right shows the corresponding constraint automaton where we assume that only bits 0 and 1 can be transmitted through the channels. The initial state stands for the configuration where the buffer is empty, while the two other states represent the configurations where the buffer is filled with one of the data items. The outgoing transitions from the initial state are labeled with the singleton set $\{A\}$ which reflects the fact that in the initial configuration only data-flow at $A$ is possible. If the buffer is filled then data-flow at $A$ is impossible and only $B$ and $C$ can take the value from the buffer.

In the sequel, we specify constraint automata using a nonempty and finite set Data consisting of data items that can be sent (and received) via channels and a nonempty and finite set $\mathcal{N}=\left\{A_{1}, \ldots, A_{n}\right\}$ of names. Intuitively, we may think of the $A_{i}$ 's to be the source or sink nodes of a Reo circuit. We refer to the subsets of $\mathcal{N}$ as node-sets.

Data assignments, data constraints. A data assignment for $\emptyset \neq N \subseteq \mathcal{N}$ is a function $\delta: N \rightarrow$ Data. DA(N) denotes the set of all data assignments for $N$, and $D A$ the set of all data assignments (on any $N$ ). Data constraints, which can be viewed as a symbolic representation of sets of data assignments, are formally defined as propositional formulas built from the atoms " $d_{A} \in P$ " and " $d_{A}=d_{B}$ ", where $A, B \in \mathcal{N}, d_{A}, d_{B} \in$ Data, and $P \subseteq$ Data. DC $(N)$ denotes the set of data constraints using only names from $N$, and $D C$ is a shorthand for $D C(\mathcal{N})$. We simply write " $d_{A}=d$ " rather than " $d_{A} \in\{d\}$ ". The symbol $\models$ stands for the obvious satisfaction relation which results from interpreting data constraints over data assignments. Satisfiability and logical equivalence $\equiv$ of data constraints are defined as usual.

Definition 3.0.2 (Constraint automata, [4]) A constraint automaton (over Data) is a tuple $\mathcal{A}=\left(Q, \mathcal{N}, \longrightarrow, Q_{0}\right)$ where $Q$ is a finite set of states, $\mathcal{N}$ a finite set of nodes, $\longrightarrow$ is a finite subset of $Q \times\left(2^{\mathcal{N}} \times D C\right) \times Q$, called the transition relation, and $Q_{0} \subseteq Q$ a nonempty set of initial states. We write $q \stackrel{N, g}{\longrightarrow} p$ instead of $(q, N, g, p) \in \longrightarrow$ and require that (1) $N \neq \emptyset$ and (2) $g \in D C(N)$ is satisfiable. We call $N$ the node-set and $g$ the guard of the transition. States without any outgoing transition are called terminal.

The intuitive meaning of a constraint automaton as an operational model for Reo connectors is similar to the interpretation of labeled transition systems as formal models for reactive systems. The sink and source nodes of a Reo connector circuit play the role of the nodes in its corresponding constraint automaton. The states represent the configurations of the connector. The meaning of a transition $q \stackrel{N, g}{\longrightarrow} p$ is that in configuration $q$ all the nodes $A_{i} \in N$ perform (synchronously) I/O-operations that meet the guard $g$, resulting in a new configuration $p$, while at the same moment there is no data-flow at the other nodes $A_{i} \in \mathcal{N} \backslash N$.

Example 3.0.3 (Constraint automata) Constraint automata for the various basic channels types, the exclusive router and shift-lossy FIFO1 channel are shown in Figure 3 (where valid guards have been omitted). The automaton for a FIFO1 channel with source A and sink B is the same as the one for the example in the beginning of the section, except that $C$ has to be removed. These automata do not have terminal states as in any configuration data flow at some nodes is possible.
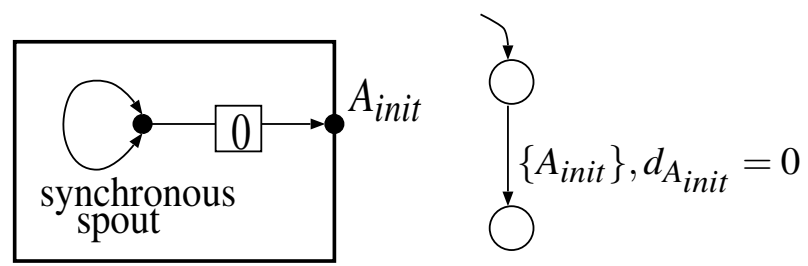

Component "Init"
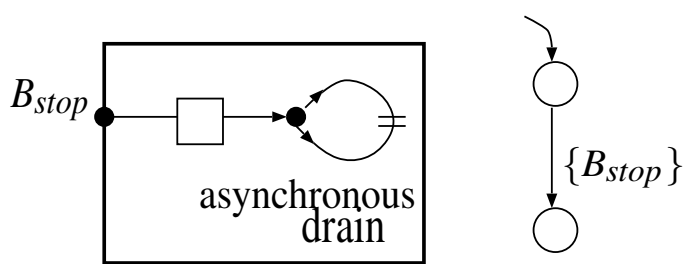

Component "Stop" 
The left part of the picture above shows the Reo circuit for an initializer, i.e., a component without input ports (source nodes) and a single output port $A_{\text {init }}$ where data-flow at $A_{\text {init }}$ happens exactly once. ${ }^{2}$ Thus, if we connect $A_{\text {init }}$ with an input port $A$ of another component $C$ via a synchronous channel with source $A_{\text {init }}$ and $\operatorname{sink} A$ then data-flow at $A_{\text {init }}$ activates the data-flow at $C$ but prevents any "restart" of $C$. The situation is similar for the component "Stop" on the right of the picture where the source node $B_{\text {stop }}$ can put a value into the buffer exactly once, because afterward the buffer is filled forever as no data-flow is possible for an asynchronous drain with both source ends coincident on the same node. Thus, if an output port $B$ of a component $C$ is connected via a synchronous channel with $B_{\text {stop }}$ then output at B is possible exactly once. In this sense, component "Stop" can serve to terminate data-flow in other components.

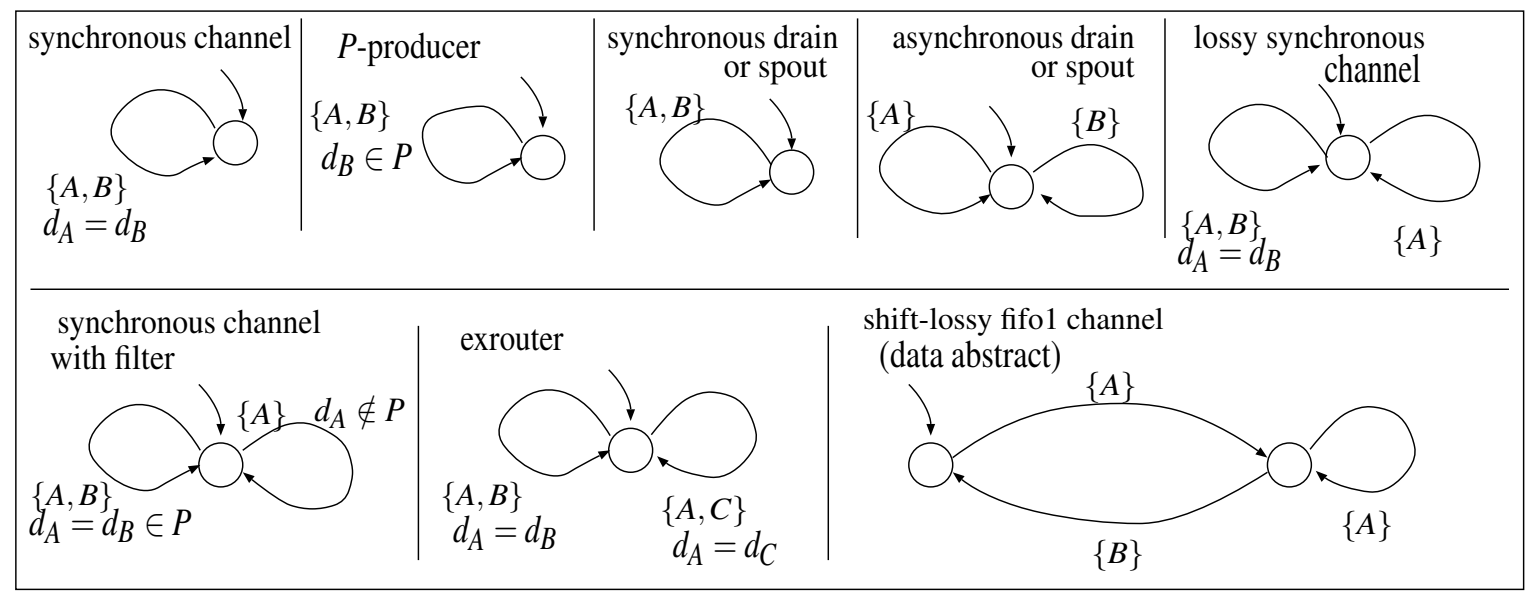

Figure 3: Constraint automata for some basic channels in Reo

In [4], we formalized the semantics of a constraint automaton as a relation on timed data streams. For the purposes of this paper, an equivalent, but simpler concept suffices which abstracts away from time and describes the "traces" of a constraint automaton by scheduled-data streams: finite or infinite sequences of pairs $\langle N, \delta\rangle$, consisting of a set $N$ of all the nodes that are scheduled to be synchronously (i.e., atomically) active in the next step, together with a data assignment $\delta \in D A(N)$ describing the data values that are input and output.

Definition 3.0.4 (Scheduled-data streams, generated language) A scheduled-data stream $\Theta$ is a finite or infinite sequence of pairs in $2^{\mathcal{X}} \times D C$, denoted by $\Theta(i)=\langle\Theta . N(i), \Theta . \delta(i)\rangle$, such that $\Theta . N(i)$ is a non-empty node-set and $\Theta . \delta(i)$ a data assignment for $\Theta . N(i)$. We write $|\Theta|$ to denote the length of $\Theta$ (which can be $\omega$ ). The empty scheduleddata stream is denoted by $\varepsilon . S D S_{\mathcal{X}}$ or briefly $S D S$ denotes the set of all scheduled-data streams.

Let $\mathcal{A}=\left(Q, \mathcal{N}, \longrightarrow, Q_{0}\right)$ be a constraint automaton, $\Theta \in S D S$ and $q$ a state in $\mathcal{A}$. A $q$-run for $\Theta$ in $\mathcal{A}$ is a path in $\mathcal{A}$

$$
\mathbf{q}=q_{0} \stackrel{N_{0}, g_{0}}{\longrightarrow} q_{1} \stackrel{N_{1}, g_{1}}{\longrightarrow} q_{2} \stackrel{N_{2}, g_{2}}{\longrightarrow} \ldots
$$

such that (1) $q_{0}=q$ and (2) either $\mathbf{q}$ and $\Theta$ are infinite or $\mathbf{q}$ consists of $|\Theta|$ transitions and ends in a terminal state and (3) $N_{i}=\Theta . N(i), \Theta . \delta(i) \models g_{i}$ for all $0 \leq i<|\Theta|$. The generated language $\mathcal{L}(\mathcal{A})$ of $\mathcal{A}$ is the set of all scheduled-data streams $\Theta \in S D S$ which have a $q_{0}$-run in $\mathcal{A}$ for some initial state $q_{0} \in Q_{0}$.

For instance, the SDS-language generated by the automaton for a synchronous channel consists of all infinite scheduled-data streams $\Theta$ with $\Theta . N(i)=\{A, B\}$ and where data assignment $\Theta . \delta(i)$ assigns the same data item to $A$ and $B$.

\footnotetext{
${ }^{2}$ Data-flow at the node on the left, where the two sink ends of a synchronous spout coincide, is never possible because on the one hand, the sink ends of the spout are obligated to produce their respective data items simultaneously, while on the other hand the merge semantics of sink/mixed nodes does not allows for simultaneous data-flow at both sink ends.
} 
In [4], we explain how an automaton for a Reo circuit can be constructed in a compositional way. (For the purpose of this paper, the details of that construction do not matter. The only thing that we use later, in Sect. 5, is that by applying the above definition to the automaton for a Reo circuit $R$, we obtain an SDS-language $\mathcal{L}(R)$ for $R$.) In what follows we show, conversely, how to construct a Reo circuit from a constraint automaton.

\section{Scheduled-data expressions}

The first step of our construction of a Reo circuit from a given automaton is to transform the automaton into an equivalent $\omega$-regular expression, a so-called scheduled-data expression. These are built by $\varepsilon$ representing the singleton SDS-language $\{\varepsilon\}$ and the atoms $\langle N, g\rangle$ where $\emptyset \neq N \subseteq \mathcal{N}$ and $g$ is a satisfiable data constraint for $N$. The SDSlanguage $\mathcal{L}(\langle N, g\rangle)$ consists of all scheduled-data streams $\Theta$ of length 1 such that $\Theta . N(0)=N$ and $\Theta . \delta(0) \models g$. Moreover, we use the standard composition operators ; (concatenation), $\cup$ (union) and the closure operators $\alpha^{\omega}$ (infinitely many repetitions) and $\alpha^{\infty}$ (finite or infinite repetitions). The formal definition of $\mathcal{L}(\alpha)$ for composite expressions is defined as for ordinary $\omega$-regular expressions and is omitted here.

Similar to the construction of a finite automaton from ordinary regular expressions (see e.g. [18]), we can assign a constraint automaton to any scheduled-data expression that generates the same SDS-language and which is linear in the size of the expression. Since this construction does not play a role in the present paper, its description is omitted. Instead, we use the reverse construction, i.e., of a scheduled-data expression for a constraint automaton. Although to do so, we may apply the standard algorithms for generating ( $\omega$-)regular expressions from automata (see e.g. [18]), we suggest here an alternative algorithm. Rather than describing the construction in general, we treat a typical example. Consider the constraint automaton as shown on the left of the following picture where $a, b, c$ are pairs of name sets with corresponding data constraints.

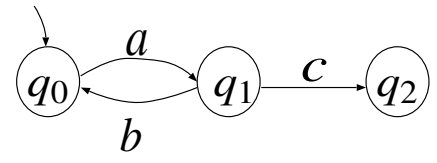

$$
\begin{aligned}
& \alpha_{0}=a ; \alpha_{1} \\
& \alpha_{1}=\left(b ; \alpha_{0}\right) \cup\left(c ; \alpha_{2}\right) \\
& \alpha_{2}=\varepsilon
\end{aligned}
$$

Let $\alpha_{i}$ denote the scheduled-data expression corresponding to (the SDS-language generated by) state $q_{i}$, for $i=$ $0,1,2$. The three transitions of this automaton give rise to three equations for the expressions as shown above. Together, they imply the following equation: $\alpha_{0}=\left(a ; b ; \alpha_{0}\right) \cup(a ; c)$. This equation can be solved, using the following general laws for scheduled-data expressions: "if $\alpha=(\beta ; \alpha) \cup \gamma$ and $\varepsilon \notin \beta$ then $\alpha=\beta^{\infty} ; \gamma$ " and "if $\alpha=\beta ; \alpha$ and $\varepsilon \notin \beta$ then $\alpha=\beta^{\omega}$ ". Applying the first law to the equation above yields the expression $\alpha_{0}=(a ; b)^{\infty} ; a ; c$ for the state $q_{0}$.

\section{From scheduled-data expressions to Reo}

We now address the question of constructing a Reo circuit for a scheduled-data expression $\alpha_{0}$. Because the source and the sink nodes of a Reo circuit play different roles with respect to its environment, and this distinction is abstracted away in scheduled-data expressions (and constraint automata), we first need to identify the "input" and "output" of a circuit by partitioning its node set $\mathcal{N}$. Let $\mathcal{N}=\left\{C_{1}, \ldots, C_{n}\right\} \cup\left\{D_{1}, \ldots, D_{m}\right\}$ contain all nodes occurring in the node-sets $N$ of the atoms $\langle N, g\rangle$ in $\alpha_{0}$, where we assume that the $C_{i}$ 's are source nodes and the $D_{j}$ 's are sink nodes. Our goal is the construction of a Reo circuit $R$ with source nodes $C_{1}, \ldots, C_{n}$ and sink nodes $D_{1}, \ldots, D_{m}$ such that $\mathcal{L}\left(\alpha_{0}\right)=\mathcal{L}(R)$.

For the construction of $R$, we use a compositional approach that builds a Reo circuit $R_{\alpha}$ for each subexpression $\alpha$ of $\alpha_{0}$. Fig. 4 shows the general structure of $R_{\alpha}$ : if the source node $A_{\alpha}$ is fed from outside with some data element, then it is put into the buffer between $A_{\alpha}$ and $\tilde{A}_{\alpha}$. As soon as $\tilde{A}_{\alpha}$ takes the data element from the buffer, the sub-circuit in the middle is "activated". Similarly, data-flow inside this sub-circuit stops as soon as a data element arrives at $\tilde{B}_{\alpha}$, which puts it into the buffer between $\tilde{B}_{\alpha}$ and $B_{\alpha}$. Thus, data-flow at the sink node $\tilde{B}_{\alpha}$ can be viewed as a signal that $R_{\alpha}$ has "terminated". 


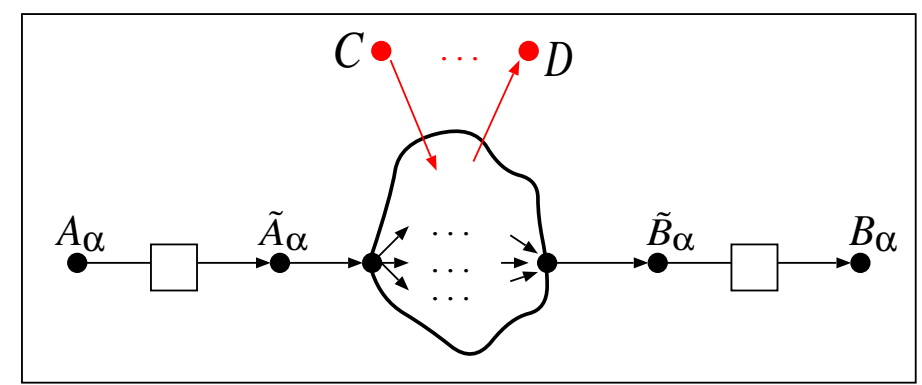

Figure 4: Structure of the Reo-circuit $R_{\alpha}$

The nodes $C$ and $D$ in Fig. 4 are there to indicate that there will be some channels connecting the sub-circuit in the middle of $R_{\alpha}$ with (some of) the source nodes $C$ and (some of) the sink nodes $D$ in $\mathcal{N}$. The construction of a circuit $R$ for an expression $\alpha_{0}$ will be completed by a last step, in which "Init" and "Stop" components, defined in Example 3.0.3, are added to begin and end the data-flow of in the circuit $R_{\alpha_{0}}$, as shown in the following picture:

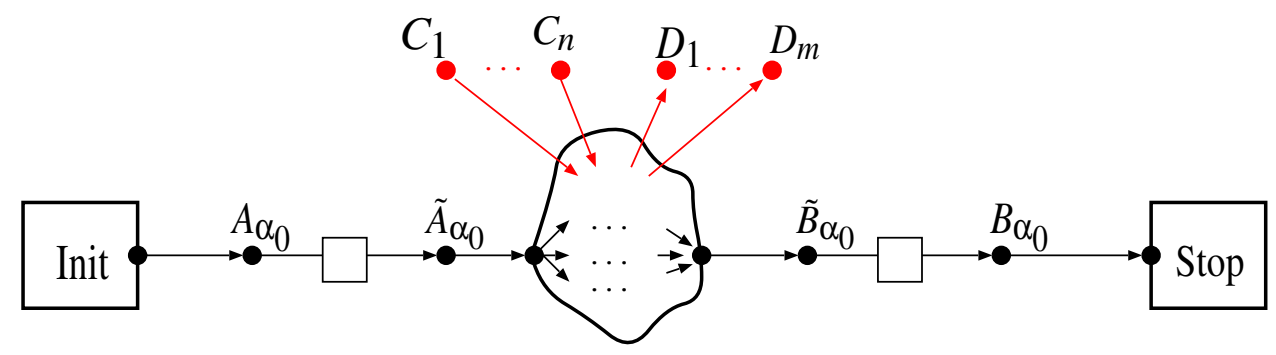

The construction of the circuit will be such that at any moment, exactly one of the leftmost and rightmost buffers or buffers inside $R_{\alpha_{0}}$ will be filled. Thus, we may consider data-flow through $R$ as a token game, where the token is passed on from left to right.

Concatenation, union and closure. We first explain how to construct a circuit $R_{\alpha}$, assuming we have already constructed the circuits for $\alpha$ 's subexpressions. (If a subexpression $\alpha$ occurs more than once in $\alpha_{0}$, e.g. if $\alpha_{0}=\alpha ; \alpha$, then we need a copy of the circuits $R_{\alpha}$ for every syntactic occurrence of $\alpha$ as a subexpression in $\alpha_{0}$.) For $\alpha=\gamma ; \beta$ the Reo circuit $R_{\alpha}$ results from combining $R_{\gamma}$ and $R_{\beta}$ as follows:

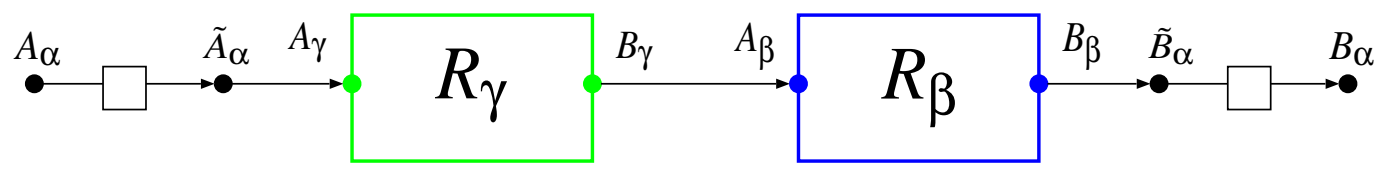

Note that the internal FIFO-channels "at the end" of $R_{\gamma}$ and "at the beginning" of $R_{\beta}$ (not drawn in the picture) ensure that in the concatenation $\gamma ; \beta$ data-flow inside $R_{\beta}$ cannot start before data-flow in $R_{\gamma}$ has finished.

For $\alpha=\gamma \cup \beta$, the Reo circuit $R_{\alpha}$ is obtained by combining $R_{\gamma}$ and $R_{\beta}$ with an exclusive router that nondeterministically chooses to "activate" the data-flow in either $R_{\gamma}$ or $R_{\beta}$ : 


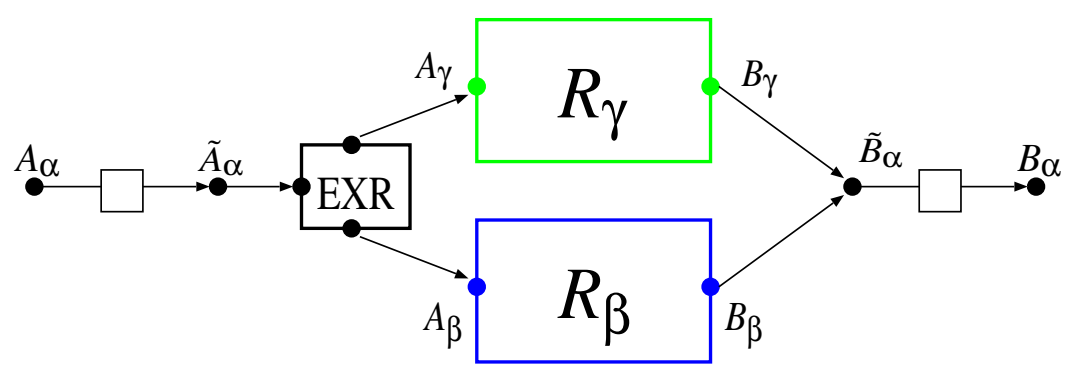

The Reo circuit $R_{\alpha}$ where $\alpha=\beta^{\infty}$ is obtained from $R_{\beta}$ as follows: ${ }^{3}$

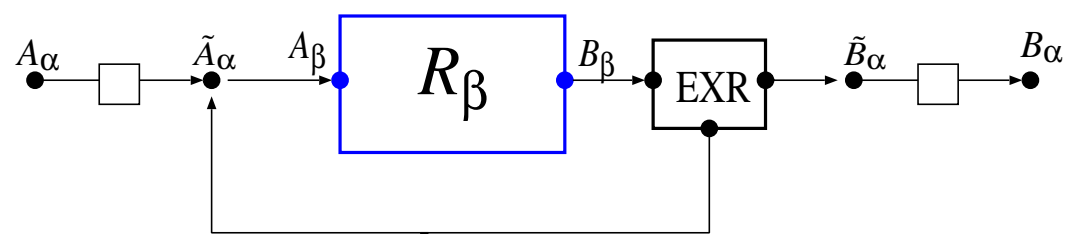

For $\alpha=\beta^{\omega}$, the Reo circuit has the following structure.

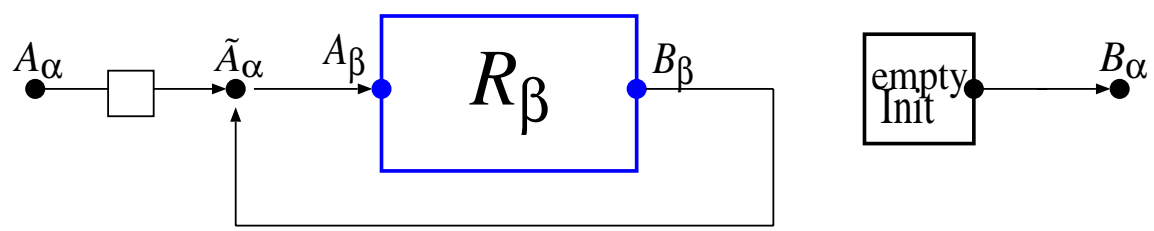

Here, "empty Init" is a variant of the initializer in Ex. 3.0.3, where the buffer is initially empty. Thus, data-flow never occurs in "empty Init" or at node $B_{\alpha}$. Being non-reachable, it may be omitted; we keep it here so that the circuit retains the general shape of Fig. 4.

The empty expression. For $\alpha=\varepsilon$, we simply use a FIFO1 channel with its source end on node $A_{\varepsilon}$ and its sink end on node $B_{\mathcal{E}}$. (Using just a single channel departs from the general schema sketched in Fig. 4, but the nodes $\tilde{A}_{\alpha}$ and $\tilde{B}_{\alpha}$ are not needed in our compositional approach.)

Atomic expressions. So far the construction of Reo circuits for composite expressions has followed patterns that are familiar from automata theory. Next we come to the most complicated and most interesting step in our construction, namely the construction of a Reo circuit for atomic expressions $\langle N, g\rangle$. The difficulty lies in the fact that such expressions model a computation step of a corresponding Reo circuit, in which certain channel ends are active and others are not. Moreover, we must ensure that at every active channel end, the right data value is input or output. Let Atoms denote the set of all atomic expressions $\langle N, g\rangle$ of $\alpha_{0}$. Recall that $N$ is a nonempty subset of $\mathcal{N}=\left\{C_{1}, \ldots, C_{n}\right\} \cup\left\{D_{1}, \ldots, D_{m}\right\}$ and $g$ is a satisfiable data constraint for the nodes in $N$. We first describe a general technique to design a Reo circuit for the atoms $\langle N, g\rangle \in$ Atoms. (Later we explain how this technique can be made more efficient in various ways.) We first transform $g$ into its canonical disjunctive normal form, which replaces it with an equivalent data constraint $h_{1} \vee \ldots \vee h_{r}$ where each of the $h$ 's is a formula of the form

$$
h=\bigwedge_{C \in N_{\mathrm{src}}}\left(d_{C} \in P_{C}\right) \wedge \bigwedge_{D \in N_{\mathrm{snk}}}\left(d_{D} \in P_{D}\right)
$$

with $N_{\text {src }}=N \cap\left\{C_{1}, \ldots, C_{n}\right\}, N_{\text {snk }}=N \cap\left\{D_{1}, \ldots, D_{m}\right\}$ and $P_{C}, P_{D} \subseteq$ Data. E.g., if $g$ is " $d_{C}=d_{D}$ " then we replace $g$ with $\bigvee_{d \in \text { Data }} h_{d}$ where $h_{d}$ is $\left(d_{C}=d\right) \wedge\left(d_{D}=d\right)$. Next, we replace $\langle N, g\rangle$ with the equivalent expression $\left\langle N, h_{1}\right\rangle \cup$ $\ldots \cup\left\langle N, h_{r}\right\rangle$, construct the circuits $R_{\left\langle N, h_{k}\right\rangle}$ (see below) and combine them with the union-operator described above.

With the formula $h$ as above, a circuit $\mathcal{R}_{\langle N, h\rangle}$ for $\langle N, h\rangle$ is presented in Fig. 5, which we now explain. For the

${ }^{3}$ The syntax of scheduled-data expressions does not include the Kleene closure $\alpha=\beta^{*}$. However, it could be treated by simply replacing the exclusive router with a fair exclusive router. 


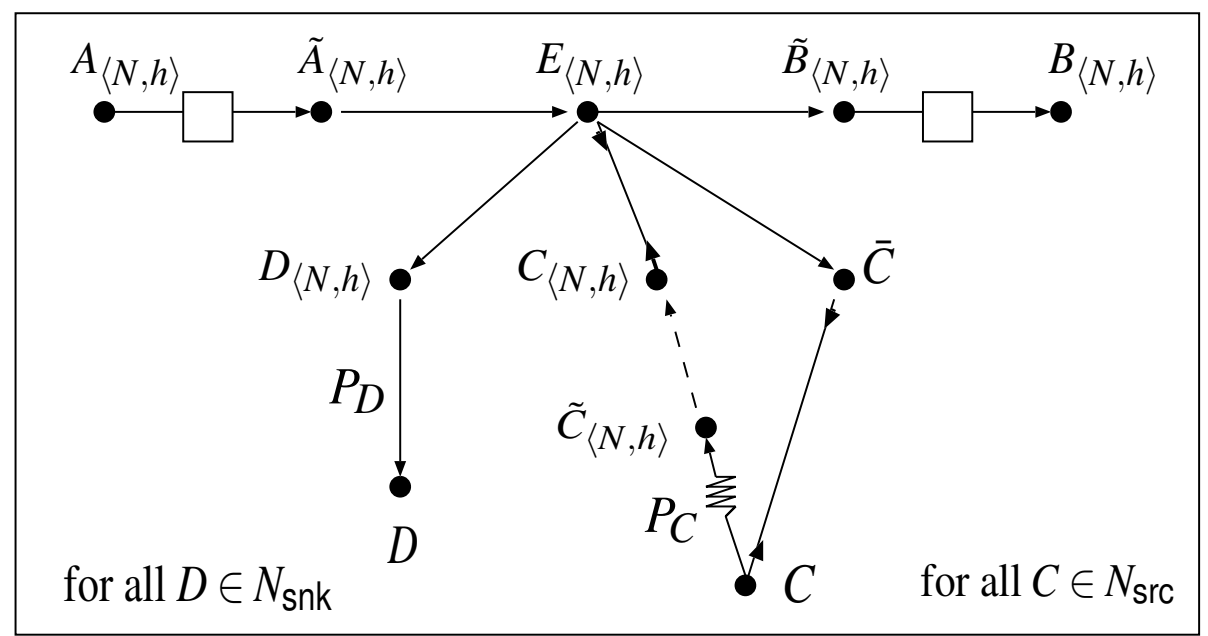

Figure 5: Reo-circuit $R_{\langle N, h\rangle}$

Reo circuit $R_{\langle N, h\rangle}$ of a given $\langle N, h\rangle$, we need a pair of nodes $C_{\langle N, h\rangle}$ and $\tilde{C}_{\langle N, h\rangle}$ for every source node $C \in N_{\mathrm{src}}$, and similarly, one node $D_{\langle N, h\rangle}$ for every sink node $D \in N_{\text {snk }}$, plus one other node $E_{\langle N, h\rangle}$. The same node $\bar{C}$ must be used for all circuits $R_{\langle M, f\rangle}$ where $C \in M$ and $\langle M, f\rangle \in$ Atoms, while the nodes $C_{\langle N, h\rangle}$ and $\tilde{C}_{\langle N, h\rangle}$ are unique for every atomic data expression $\langle N, h\rangle \in$ Atoms where $C \in N$.

We can think of the node $E_{\langle N, h\rangle}$ as a switch that synchronizes the data-flow in the upper sub-circuit with the nodes $D_{\langle N, h\rangle}, D$, and $C_{\langle N, h\rangle}, \tilde{C}_{\langle N, h\rangle}, \bar{C}$, and $C$ for all source nodes $C \in N_{\text {src }}$ and all sink nodes $D \in N_{\text {snk. The synchronous }}$ channel from $E_{\langle N, h\rangle}$ to $D_{\langle N, h\rangle}$ and the $P_{D}$-producer connecting $D_{\langle N, h\rangle}$ with $D$ ensure that any data-flow at $E_{\langle N, h\rangle}$ is synchronized with the receipt of a value $d \in P_{D}$ at sink node $D$.

For the source nodes $C$, the situation is a bit more complicated because we must ensure that $C$ accepts an input value iff $C$ synchronizes with exactly one of the nodes $E_{\langle M, f\rangle}$ where $\langle M, f\rangle$ is a subexpression of $\alpha_{0}$ with $C \in M$. The use of perfect synchronous channels is not appropriate because of the replicator semantics of the source nodes. If $C$ were connected with $E_{\langle N, h\rangle}$ via perfect synchronous channels only, then data-flow would block when $C$ appears in two or more atomic subexpressions of $\alpha_{0}$. (Note that simultaneous data-flow at different nodes $E_{\langle N, h\rangle}, E_{\langle M, f\rangle}$ is not possible.) For this reason, we connect $C$ with $E_{\langle N, h\rangle}$ via a filter channel, a lossy synchronous channel and a synchronous drain through the nodes $C_{\langle N, h\rangle}$ and $\tilde{C}_{\langle N, h\rangle}$. These three channels (1) allow $C$ to pass values even when $E_{\langle N, h\rangle}$ is not available to synchronize with $C$, and (2) force $C$ to pass a value $d \in P_{C}$ when it synchronizes with $E_{\langle N, h\rangle}$. To prevent $C$ from passing a value without synchronizing with one of the nodes $E_{\langle M, f\rangle}$ where $C \in M$, we use a synchronous channel connecting $E_{\langle N, h\rangle}$ with $\bar{C}$ and a synchronous drain between $\bar{C}$ and $C$. These channels ensure that for $C \in M, C$ is active exactly when data-flow occurs at $\bar{C}$ and exactly one of the nodes $E_{\langle M, f\rangle}$.

Size of the constructed circuit. In the worst case, the treatment of the atoms $\langle N, g\rangle$ leads to an exponential blow-up (because every disjunctive normal form for $g$ may be exponentially longer than $g$ ). However, when we assume that all data constraints in $\alpha_{0}$ are given in canonical disjunctive normal form and when we measure the length of $\alpha_{0}$ as the total length of all data constraints occurring in (one of the atoms in) $\alpha_{0}$ then the total number of channels in the constructed circuit is linear in the length of $\alpha_{0}$.

Preprocessing. We now explain how a preprocessing phase of the set Atoms can simplify the construction of the circuits for the atomic subexpressions of $\alpha_{0}$. We first look for pairs $\langle C, D\rangle$ with $C \in\left\{C_{1}, \ldots, C_{n}\right\}, D \in\left\{D_{1}, \ldots, D_{m}\right\}$ such that for all $\langle N, g\rangle \in$ Atoms either $\{C, D\} \cap N=\emptyset$ or $\{C, D\} \subseteq N$ and $g \leq d_{C}=d_{D}$. ( $\leq$ denotes logical implication.) Then, we establish a synchronous channel with its source end on node $C$, its sink end on node $D$ and remove $D$ in the sense that any $\langle N, g\rangle \in$ Atoms is replaced with $\left\langle N \backslash\{D\}, g\left[d_{D} / d_{C}\right]\right\rangle$ where $g\left[d_{D} / d_{C}\right]$ means the data constraint resulting from $g$ by the syntactic replacement of any occurrence of $d_{D}$ with $d_{C}$. Second, for any pair $\left(C_{i}, C_{j}\right)$ of source nodes such that for all $\langle N, g\rangle \in$ Atoms either $\left\{C_{i}, C_{j}\right\} \cap N=\emptyset$ or $\left\{C_{i}, C_{j}\right\} \subseteq N$ and $d_{C_{j}}$ does not occur in $g$, we establish a synchronous drain connecting $C_{i}$ and $C_{j}$ and remove $C_{j}$ from Atoms. The same technique can be applied to sink nodes $D_{i}, D_{j}$ such that for all $\langle N, g\rangle \in$ Atoms either $\left\{D_{i}, D_{j}\right\} \cap N=\emptyset$ or $\left\{D_{i}, D_{j}\right\} \subseteq N$ and $d_{D_{j}}$ does not occur 
in $g$, where we generate a synchronous spout with its sink ends $D_{i}$ and $D_{j}$ and remove $D_{j}$. Finally, we look for sink nodes $D_{i}, D_{j}$ such that $\langle N, g\rangle \in$ Atoms implies $\left\{D_{i}, D_{j}\right\} \cap N=\emptyset$ or $\left\{D_{i}, D_{j}\right\} \subseteq N$ and $g \leq d_{D_{i}}=d_{D_{j}}$ and insert a new sink node $D_{i j}$ with synchronous channels from $D_{i j}$ to $D_{i}$ and $D_{j}$. We then remove $D_{i}, D_{j}$ from Atoms and treat $D_{i j}$ as a sink node. A similar transformation $\left\langle C_{i}, C_{j}\right\rangle \rightsquigarrow C_{i j}$ applies to source nodes with such that for all $\langle N, g\rangle \in$ Atoms either $\left\{C_{i}, C_{j}\right\} \cap N=\emptyset$ or $\left\{C_{i}, C_{j}\right\} \subseteq N$ and $g \leq d_{C_{i}}=d_{C_{j}}$. However, here we need a Reo connector that checks the equality of two (synchronously) arriving input values.

Optimization. As in other algorithmic constructions, our resulting Reo circuits contain certain redundancies which can be optimized away. We can detect and remove them by applying circuit transformation rules that look for recognizable patterns of (sub-)circuits and replace them with their simpler equivalents. E.g., every occurrence of a synchronous channel preceding or following any other channel $X$ can be simplified to only $X$. An example is provided in the appendix.

\section{Conclusion}

The main contribution of the present paper is a general construction of a Reo circuit from a constraint automaton. Although similar constructions exist in the classical area of automata and digital circuits, the situation here is far more complicated because of two major differences:

1. The behaviour specified by constrained automata is generally not functional (from input to output) but relational.

2. In a digital circuit and the Mealy automaton describing it, behaviour is always synchronous. In contrast, in Reo, behaviour can be synchronous, asynchronous, or (at different steps) a combination of the two.

Because of in particular point 2, the classical construction of a circuit from an automaton breaks down, and at forehand, it was by no means obvious how to tackle the problem for Reo. We see the algorithm described in the present paper therefore as a major step forward in the automatic synthesis of Reo component connector circuits.

Although the construction presented here is not overly complicated, it can and should still be simplified further and made more efficient. Parts of such considerations have already been sketched in the present paper.

A compositional approach similar to the one we suggest here can also be used to encode in Reo processes specified by terms of CCS- or CSP-like process algebras. In fact, some of the typical operators are already included in regular expressions (CCS-like nondeterminism corresponds to union, sequential composition to concatenation and $\varepsilon$ to, e.g., the CCS-process nil). Parallel composition with CCS- or CSP-like synchronization can be realized by establishing appropriate synchronous channels and Reo's join operator. A LOTOS-like disrupt operator $P[>Q$ can be obtained using a Reo component that realizes a switch; this switch is initially "on" and synchronizes with $P$ as long as it is "on" but is turned "off" by $Q$ 's first activity.

In our future work, we will investigate further optimizations and the design of an alternative synthesis algorithm that goes directly from automata to Reo circuits without having the regular expressions as an intermediate step. Furthermore, dynamic reconfiguration of connector circuits is an inherent aspect of Reo that we plan to cover in our future work. 


\section{References}

[1] M. Abadi, L. Lamport, and P. Wolper. Realizable and unrealizable concurrent program specifications. In Proc. 16th International Colloquium on Automata, Languages, and Programming (ICALP), volume 372 of Lecture Notes in Computer Science, pages 1-17, 1989.

[2] F. Arbab. Abstract behavior types: A foundation model for components and their composition. In [12], pages 33-70, 2003.

[3] F. Arbab. Reo: A channel-based coordination model for component composition. Mathematical Structures in Computer Science, 14(3):1-38, 2004.

[4] F. Arbab, C. Baier, J.J.M.M. Rutten, and M. Sirjani. Modeling component connectors in reo by constraint automata. In FOCLASA'03, Electronic Notes in Theoretical Computer Science, To appear 2003. Full version see http://web.informatik.uni-bonn.de///baier/publikationen.html.

[5] F. Arbab and J.J.M.M. Rutten. A coinductive calculus of component connectors. In D. Pattinson M. Wirsing and R. Hennicker, editors, Recent Trends in Algebraic Development Techniques, Proceedings of 16th International Workshop on Algebraic Development Techniques (WADT 2002), volume 2755 of Lecture Notes in Computer Science, pages 35-56. Springer-Verlag, 2003. http://www.cwi.nl/ftp/CWIreports/SEN/SEN-R0216.pdf.

[6] A. Arora, P.C. Attie, and E.A. Emerson. Synthesis of fault-tolerant concurrent programs. In Proc. Principles of Distributed Computing, ACM Press, pages 173-182, 1998.

[7] P.C. Attie and E.A. Emerson. Synthesis of concurrent systems with many similar sequential processes. In Proc. 16th Symposium on Principles of Programming Languages, ACM Press, pages 191-201, 1989.

[8] M. Broy and K. Stolen. Specification and Development of Interactive Systems: Focus on Streams, Interfaces and Refinement. Springer-Verlag, 2000.

[9] J.R. Büchi and L.H. Landweber. Solving sequential conditions by finite-state strategies. Transactions of the American Mathematical Society, 138:295-311, 1969.

[10] A. Church. Logic, arithmetic and automata. In Proc. International Congress of Mathematicians, pages 23-35. Institut Mittag-Leffler, 1962.

[11] CIM. http://www.almende.com/cim/.

[12] F.S. de Boer, M.M. Bonsangue, S. Graf, and W.-P. de Roever, editors. Formal Methods for Components and Objects, volume 2852 of Lecture Notes in Computer Science. Springer, 2003.

[13] N. Diakov and F. Arbab. Compositional construction of web services using Reo. In Proc. International Workshop on Web Services: Modeling, Architecture and Infrastructure (ICEIS 2004), Porto, Portugal, April 13-14, 2004.

[14] D. Dill. Trace Theory for Automatic Hierachical Verification of Speed Independent Circuits. MIT Press, 1989.

[15] E.A. Emerson and E.M. Clarke. Using branching time logic to synthesize synchronous skeleton. Science of Programming, 2:241-266, 1982.

[16] T. Henzinger, R. Jhala, and R. Majumdar. Counterexample-guided control. In Proc. 30th International Colloquium on Automata, Languages, and Programming (ICALP), volume 2719 of Lecture Notes in Computer Science, pages 886-902, 2003.

[17] T. Henzinger, S. Krishnan, O. Kupferman, and F. Mang. Synthesis of uninitialized systems. In Proceedings of the 29th International Colloquium on Automata, Languages, and Programming (ICALP), volume 30 of Lecture Notes in Computer Science, pages 644-656. Springer-Verlag, 2002.

[18] J. E. Hopcroft, R. Motwani, and J. D. Ullman. Introduction to Automata Theory, Language, and Computation. Addison-Wesley, 2nd edition edition, 2001.

[19] O. Kupferman and M. Vardi. Church's problem revisited. In The Bulletin of Symbolic Logic, volume 5, pages 245-263, 1999. 
[20] Z. Manna and P. Wolper. Synthesis of communicating processes from temporal logic specifications. ACM Transactions on Programming Languages and Systems, 6:68-93, 1984.

[21] P. Panangaden and F. van Breugel, editors. Mathematical Techniques for Analyzing Concurrent and Probabilistic Systems. CRM Monograph Series. American Mathematical Society, 2004. ISSN: 1065-8599.

[22] A. Pnueli and R. Rosner. On the synthesis of a reactive module. In Proc. 16th Symposium on Principles of Programming Languages, pages 179-190. ACM Press, 1989.

[23] M. Rabin. Automata on infinite objects and church's problem. Regional Conference Series in Mathematics, 13, 1972.

[24] P.J. Ramadge and W.M. Wonham. The control of discrete event systems. In Proceedings of IEEE, 77(1), pages 81-98, 1989.

[25] J.J.M.M. Rutten. Component connectors. In [21], chapter 5, pages 73-87. 2004.

[26] W. Thomas. On the synthesis of strategies in infinite games. In Proc. of the 12th Annual Symp. on Theoretical Aspects of Computer Science, volume 900 of LNCS, pages 1-13, 1995.

[27] M. Vardi. An automata-theoretic approach to fair realizability and synthesis. In Proc. CAV, volume 939 of Lecture Notes in Computer Science, pages 267-278, 1995. 


\section{A Example}

A concrete example for the Reo-circuit which is constructed from the scheduled-data expression $\alpha=\langle N, h\rangle \cup\langle M, f\rangle$ is shown in Fig. 6. Here, we assume that $N=\{C, D\}, M=\{C\}$ and $h$ is $\left(d_{C} \in P_{C}\right) \wedge\left(d_{D} \in P_{D}\right)$ while $f$ is $d_{C} \in T_{C}$. In Fig. 6, there are multiple candidates that qualify for the application of the circuit transformation rule mentioned in the end of Section 5. There are more specialized patterns that are applicable only in the context of our resulting circuits. For instance, we know that in Fig. 6, data-flow can occur through only one of the top or bottom branches of the circuit (because there is only one token at a time that passes through the entire circuit; the exclusive router; and because the two branches are isolated from one another by drains). This makes the right-hand-side FIFO1 channels on both top and bottom branches redundant.

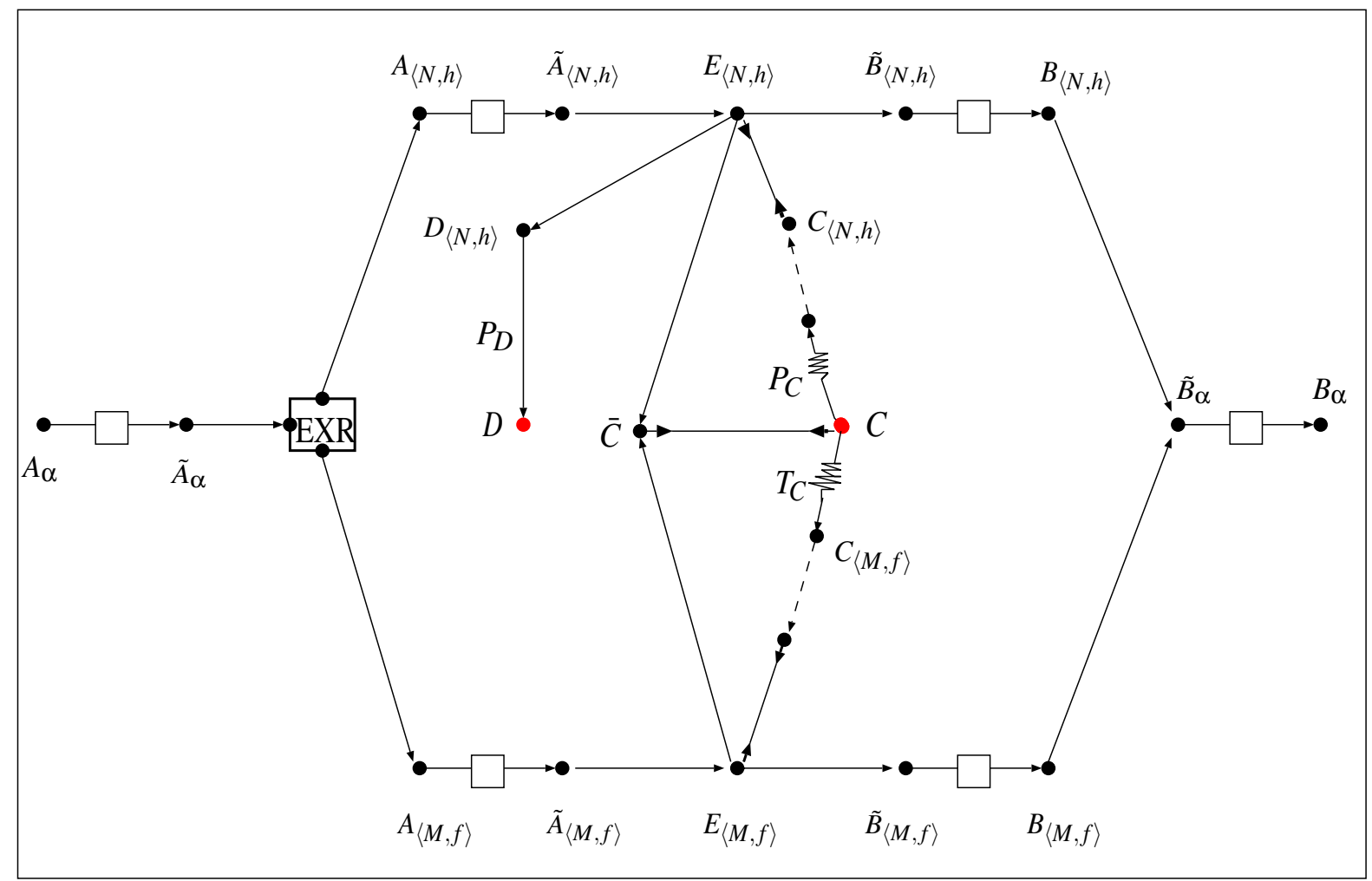

Figure 6: Reo circuit $R_{\alpha}$ for $\alpha=\langle N, h\rangle \cup\langle M, f\rangle$ where $N=\{C, D\}, M=\{C\}$ 


\section{B Correctness of construction}

We now provide a proof sketch for the soundness of our algorithm. That is, we have to show that the final circuit $R_{\alpha_{0}}^{+}$ for scheduled-data expression $\alpha_{0}$ generates the SDS-language $\mathcal{L}\left(\alpha_{0}\right)$. Recall that $R_{\alpha_{0}}^{+}$arises from $R_{\alpha_{0}}$ by adding an initializer and terminator as shown in the beginning of Section 5.

Extended circuits. The crucial point of our construction is that the circuits $R_{\alpha}$ as in Fig. 4 generate exactly the scheduled-data streams in $\mathcal{L}(\alpha)$, provided there is exactly one input at $A_{\alpha}$ and exactly one output at $B_{\alpha}$. Moreover, $R_{\alpha}$ returns to its initial configuration after the output at $B_{\alpha}$. The latter condition is essential for the treatment of the closure-operators $\alpha=\beta^{\omega}$ or $\alpha=\beta^{\infty}$.

For this reason, we argument with an extension of the circuits $R_{\alpha}$ (for the subexpressions $\alpha$ of $\alpha_{0}$ ) by a FIFO1 channel with source end on $B_{\alpha}$ and sink end on $A_{\alpha}$ as shown in Fig. 7. This circuit is called ext $\left(R_{\alpha}\right)$. To be able to

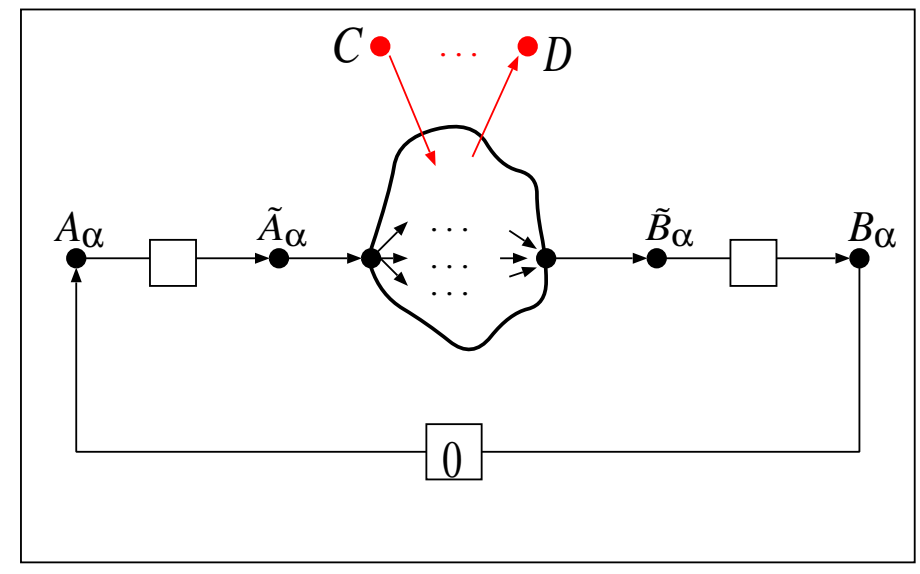

Figure 7: $\operatorname{ext}\left(R_{\alpha}\right)$ : extension of the Reo-circuit $R_{\alpha}$

reason about data flow at nodes $A_{\beta}, B_{\beta}$ for $\alpha=\beta$ and the proper subexpressions of $\alpha$ we assume that all nodes (sink, source and mixed nodes) are observable. Thus, the underlying node-set of ext $\left(R_{\alpha}\right)$ and its constraint automaton is

$$
\begin{aligned}
\mathcal{N}_{\alpha}= & \mathcal{N} \cup\left\{A_{\beta}, B_{\beta}, \tilde{A}_{\beta}, \tilde{B}_{\beta}: \beta \text { is a subexpression of } \alpha\right\} \\
& \cup \bigcup_{\langle N, h\rangle}\left(\left\{E_{\langle N, h\rangle}\right\} \cup \bigcup_{C \in N_{\mathrm{src}}}\left\{C_{\langle N, h\rangle}, \tilde{C}_{\langle N, h\rangle}, \bar{C}\right\} \cup \bigcup_{D \in N_{\mathrm{snk}}}\left\{D_{\alpha}\right\}\right)
\end{aligned}
$$

where $\langle N, h\rangle$ ranges over all atoms occurring in $\alpha$. (Recall that $N_{\text {src }}=N \cap\left\{C_{1}, \ldots, C_{n}\right\}$ and $N_{\text {snk }}=N \cap\left\{D_{1}, \ldots, D_{m}\right\}$.)

Constraint automata for the extended Reo circuits. The constraint automaton for ext $\left(R_{\alpha}\right)$ has the form as shown in Fig. 8. $\tilde{\mathcal{A}}_{\alpha}$ is a sub-automaton with initial state $\tilde{q}_{\alpha}$ and node-set $\tilde{\mathcal{N}}_{\alpha}=\mathcal{N}_{\alpha} \backslash\left\{A_{\alpha}, B_{\alpha}\right\}$. None of the state inside $\tilde{A}_{\alpha}$ can reach $q_{\alpha}$. Moreover, we have:

(1) $q_{\alpha}$ is the initial configuration of $\operatorname{ext}\left(R_{\alpha}\right)$ where the new buffer connecting $B_{\alpha}$ and $A_{\alpha}$ contains data item 0 , while all other buffers are empty.

(2) If $\alpha \neq \varepsilon$ then $\tilde{q}_{\alpha}$ is the configuration of $\operatorname{ext}\left(R_{\alpha}\right)$ where the buffer connecting $A_{\alpha}$ and $\tilde{A}_{\alpha}$ contains data item 0 , while all other buffers are empty. ${ }^{4}$

(3) $\tilde{p}_{\alpha}$ is the configuration of $\operatorname{ext}\left(R_{\alpha}\right)$ where the buffer connecting $\tilde{B}_{\alpha}$ and $B_{\alpha}$ contains data item 0 , while all other buffers are empty.

\footnotetext{
${ }^{4}$ For the empty expression $\boldsymbol{\varepsilon}$, this buffer does not exist. In that case, $\tilde{\mathcal{A}} \mathcal{E}$ consists of a single state $\tilde{q} \mathcal{E}=\tilde{p} \mathcal{E}$.
} 


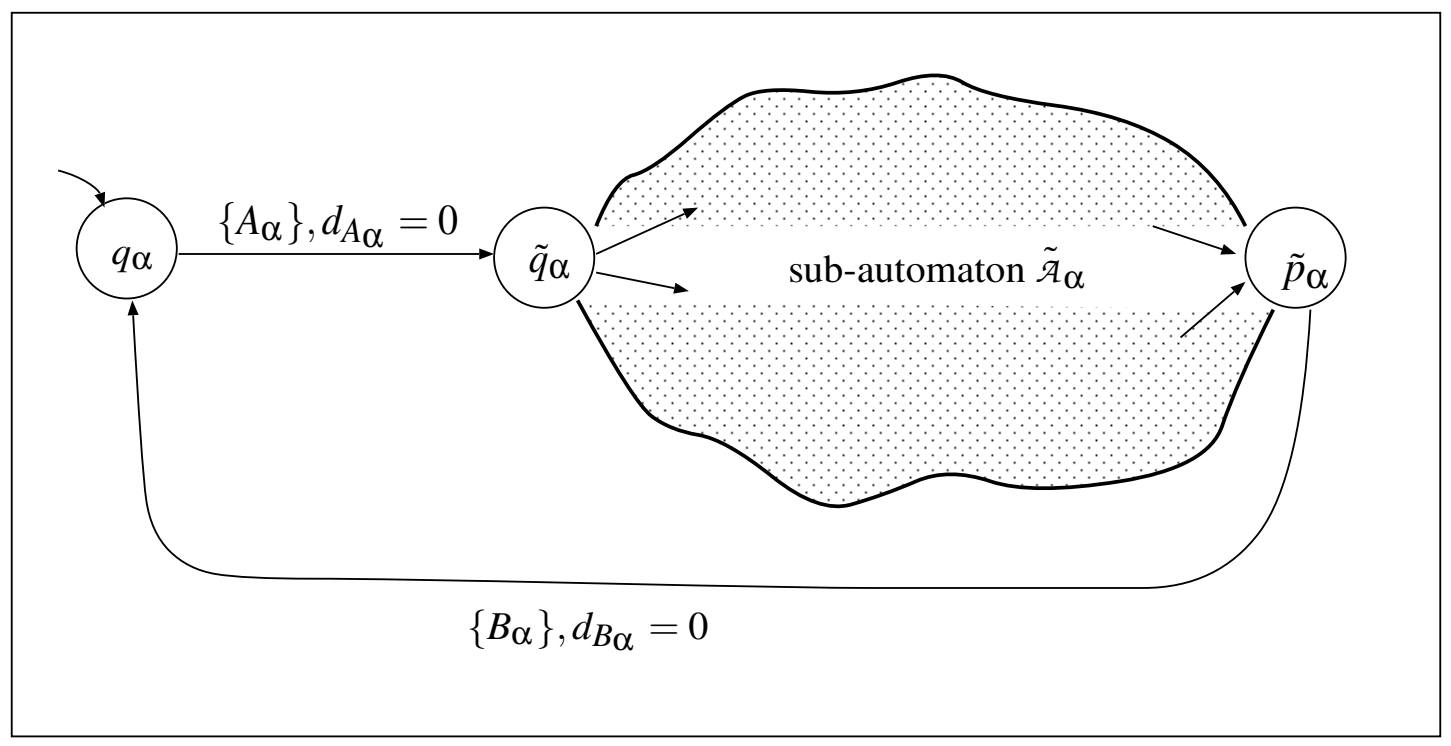

Figure 8: Constraint automaton for $\operatorname{ext}\left(R_{\alpha}\right)$

(4) The generated SDS-language $\mathcal{L}\left(\operatorname{ext}\left(R_{\alpha}\right)\right)$ over the node-set $\mathcal{N}_{\alpha}$ can be described by the scheduled-data expression

$$
\left(\left\langle\left\{A_{\alpha}\right\}, d_{A_{\alpha}}=0\right\rangle ; \mathcal{L}\left(\tilde{\mathcal{A}}_{\alpha}\right) ;\left\langle\left\{B_{\alpha}\right\}, d_{B_{\alpha}}=0\right\rangle\right)^{\omega}
$$

where $\mathcal{L}\left(\tilde{\mathcal{A}}_{\alpha}\right)$ stands for a scheduled-data expression that characterizes the accepted SDS-language of the sub-automaton $\tilde{\mathcal{A}}_{\alpha}$. with node-set $\tilde{\mathfrak{N}}_{\alpha}$.

To prove this, we may use an inductive argument. The case $\alpha=\langle N, h\rangle$ where $h$ is as in Section 5 can be verified using the join-operator $\bowtie$ that has been introduced in [4]. In essential, $\bowtie$ is a product construction that allows for the compositional construction of automata for given Reo circuits. Applying the join operator to all channels that are involved in $\operatorname{ext}\left(R_{\alpha}\right)$ we obtain the following automaton: ${ }^{5}$

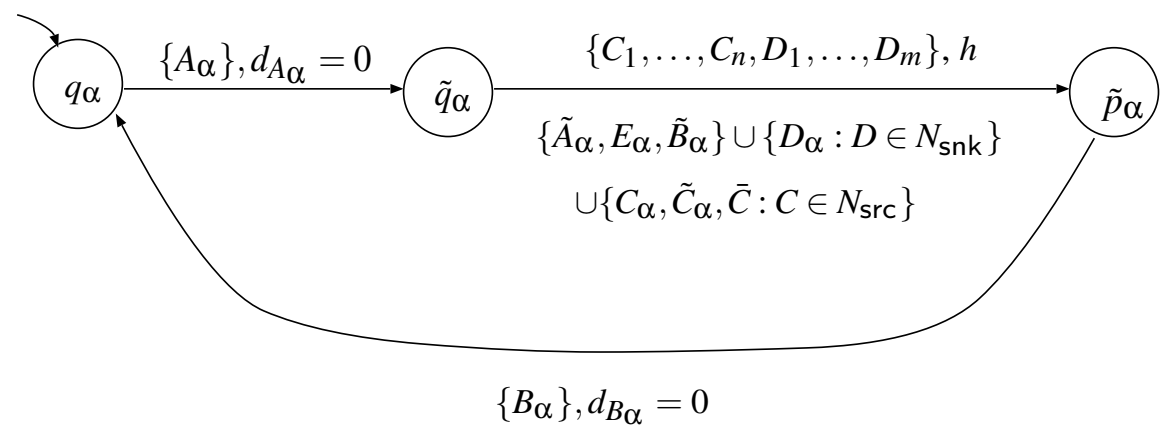

We will not consider all details of the construction of the automaton by joining the automata for all involved channels. To give an impression how the construction works, Fig. 9 illustrates the most difficult case of joining the channels that are involved in the subcircuit for a source node $C \in N$.

For $\alpha=\varepsilon$, the extended Reo circuit and its automaton have the following structure:

\footnotetext{
${ }^{5}$ We skip the data constraints concerning the additional nodes $E_{\alpha}, \tilde{A}_{\alpha}$, etc.
} 

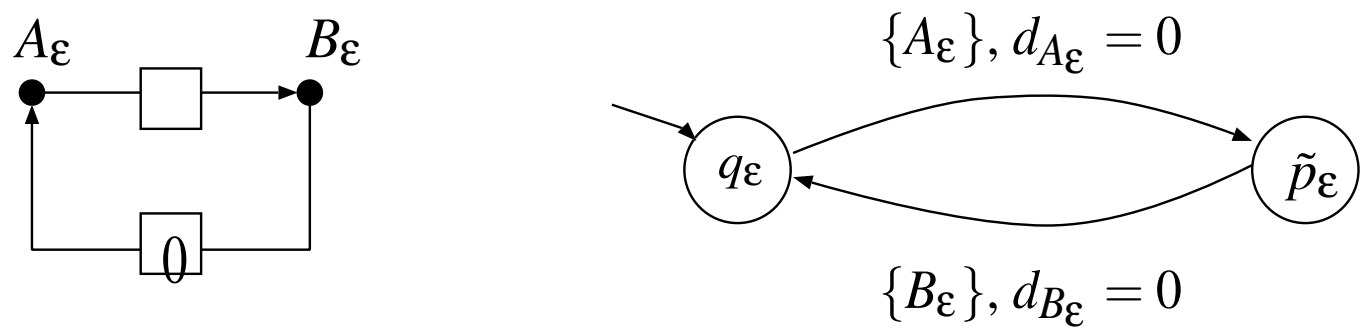

That is, here, the sub-automaton $\tilde{\mathcal{A}}_{\mathcal{E}}$ consists of a single state $\tilde{q}_{\mathcal{\varepsilon}}=\tilde{p}_{\mathcal{E}}$.

In the induction step $\alpha \in\left\{\gamma ; \beta, \beta^{\infty}, \beta^{\omega}\right\}$ we may again use the join-operator to verify the structure of the resulting automaton and the properties (1)-(4). Conditions (1) and (3) allow to reason about the automaton for the original circuits, without the additional FIFO1 channel between $B_{\beta}$ and $A_{\beta}$ for the subexpressions $\beta$ of $\alpha$. Another crucial point in the argumentation for union or concatenation is that when combining the automaton for the two sub-circuits $R_{\gamma}$ and $R_{\beta}$ then there is no undesired interference between the common sink nodes $D$ or source nodes $C$. We will skip the details here and just illustrate in Fig. 10 the situation where a source node $C$ occurs in atoms $\langle N, h\rangle$ of $\gamma$ and $\langle M, f\rangle$ of $\beta$. Moreover, here we assume that $h$ contains the sub-constraint " $d_{C} \in P_{C}$ " and $f$ the sub-constraint " $d_{C} \in T_{C}$ ". The picture on the right shows the constraint automaton that is obtained for the sub-circuit shown on the left. Intuitively, the picture for the constraint automaton makes clear that there are two transitions representing the cases where $C$ performs a write operation: one for the atom $\langle N, h\rangle$ in $\gamma$, one for the atom $\langle M, f\rangle$ in $\beta$.

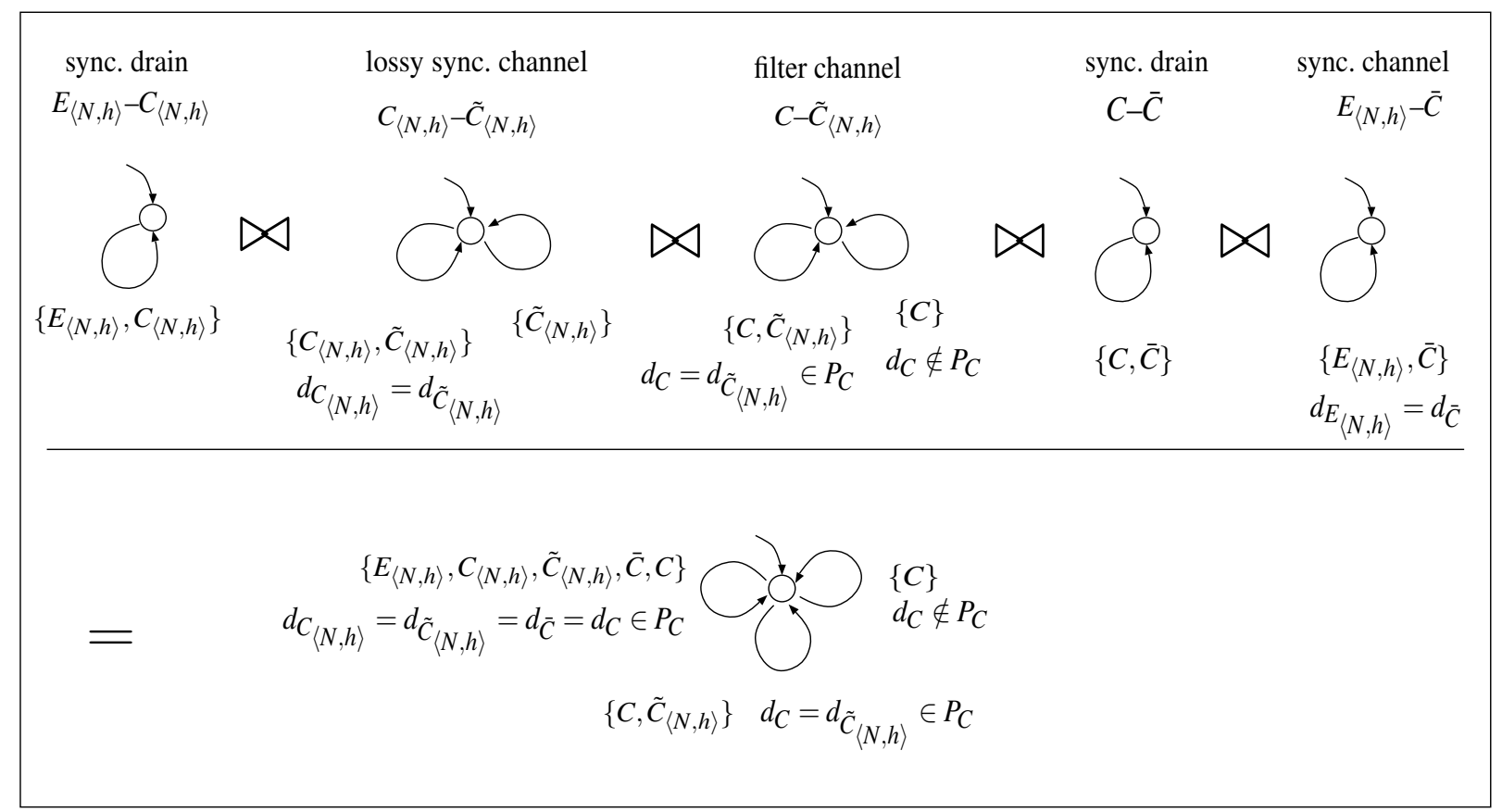

Figure 9: Constraint automata for the channels between $E_{\langle N, h\rangle}, C_{\langle N, h\rangle}, \tilde{C}_{\langle N, h\rangle}, \bar{C}$ and $C$

Extended scheduled-data expressions. We now do a similar extension with the scheduled-data expressions and switch from $\alpha$ to the expression $\operatorname{ext}(\alpha)$ which is defined by structural induction:

$$
\begin{array}{ll}
\operatorname{ext}(\boldsymbol{\varepsilon}) & =\left\langle\left\{A_{\boldsymbol{\varepsilon}}\right\}, \text { true }\right\rangle ;\langle\{B \boldsymbol{\varepsilon}\}, \text { true }\rangle \\
\operatorname{ext}(\langle N, g\rangle) & =\left\langle\left\{A_{\langle N, g\rangle}\right\}, \text { true }\right\rangle ;\langle N, g\rangle ;\left\langle\left\{B_{\langle N, g\rangle}\right\}, \text { true }\right\rangle \\
\operatorname{ext}(\gamma ; \beta) & =\left\langle\left\{A_{\gamma ; \beta}\right\}, \text { true }\right) ; \operatorname{ext}(\gamma) ; \operatorname{ext}(\beta) ;\left\{\left[B_{\gamma ; \beta}\right\}, \text { true }\right\rangle \\
\operatorname{ext}(\gamma \cup \beta) & =\left\langle\left\{A_{\gamma \cup \beta}\right\}, \text { true }\right\rangle ;(\operatorname{ext}(\gamma) \cup \operatorname{ext}(\beta)) ;\left\langle\left\{B_{\gamma \cup \beta}\right\}, \text { true }\right\rangle \\
\operatorname{ext}\left(\beta^{\omega}\right) & =\left\langle\left\{A_{\beta^{\omega}}\right\}, \text { true }\right\rangle ;(\operatorname{ext}(\beta))^{\omega} ;\left\langle\left\{B_{\beta^{\omega}}\right\}, \text { true }\right\rangle \\
\operatorname{ext}\left(\beta^{\infty}\right) & =\left\langle\left\{A_{\beta^{\infty}}\right\}, \text { true }\right\rangle ;(\operatorname{ext}(\beta))^{\infty} ;\left\langle\left\{B_{\beta^{\infty}}\right\}, \text { true }\right\rangle
\end{array}
$$



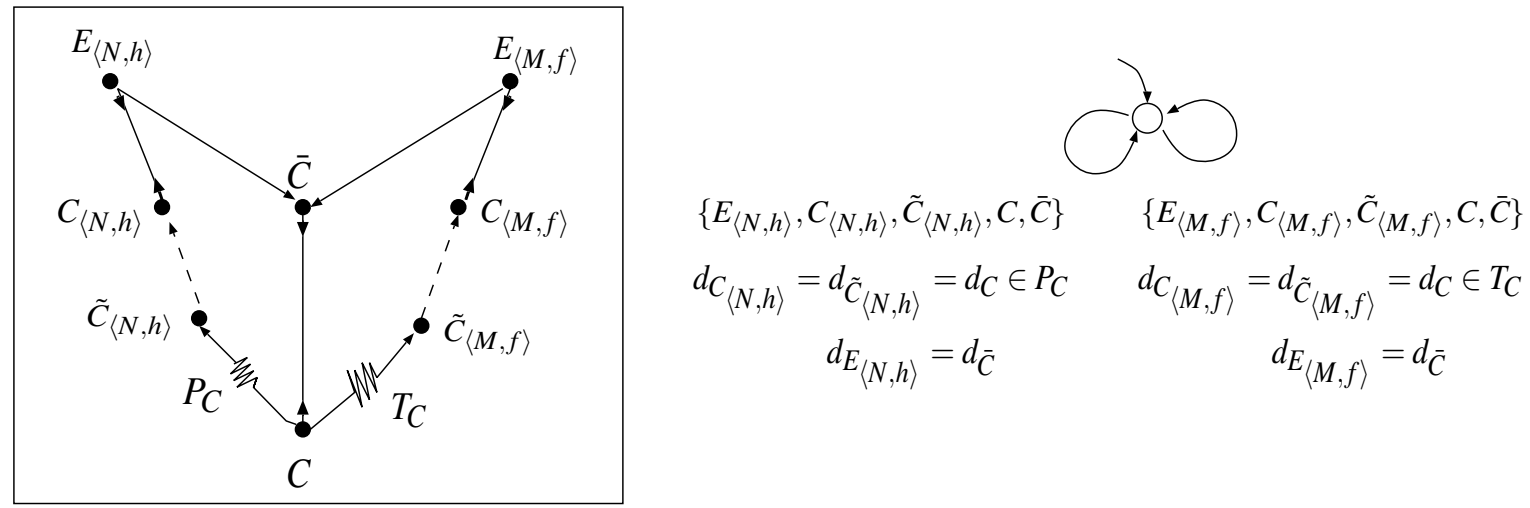

Figure 10: "Relevant sub-circuit" of $R_{\alpha}$ for source node $C$ occurring in two atoms $\langle N, h\rangle$ and $\langle M, f\rangle$

The relation between the SDS-languages of $\alpha$ and $\operatorname{ext}(\alpha)$ is as follows:

$$
\left.\mathcal{L}(\operatorname{ext}(\alpha))\right|_{\mathfrak{K}}=\mathcal{L}(\alpha)
$$

where $\left.L\right|_{\mathscr{C}}$ denotes the restriction of SDS-language $L$ to the node-set $\mathcal{N}=\left\{C_{1}, \ldots, C_{n}, D_{1}, \ldots, D_{m}\right\}$. Formally, let $\mathcal{M}$ and $\mathcal{K}$ be node-sets with $\mathcal{K} \subseteq \mathcal{M}$ and $L \subseteq S D S_{\mathcal{M}}$ then

$$
\left.L\right|_{\mathcal{K}}=\left\{\left.\Theta\right|_{\mathcal{K}}: \Theta \in L\right\}
$$

where for a scheduled-data stream $\Theta \in S D S_{\mathcal{M}}$, the scheduled-data stream $\left.\Theta\right|_{\mathcal{K}}$ results from $\Theta$ by (i) removing all pairs $\Theta(i)=\left\langle N_{i}, \delta_{i}\right\rangle$ where $N_{i} \cap \mathcal{K}=\emptyset$ and (ii) replacing any remaining pair $\Theta(i)=\left\langle N_{i}, \delta_{i}\right\rangle$ with $\left\langle N_{i} \cap \mathcal{K},\left.\delta_{i}\right|_{N_{i} \cap \mathcal{K}}\right\rangle$ where $\left.\delta_{i}\right|_{N_{i} \cap \mathcal{K}} \in D A\left(N_{i} \cap \mathcal{N}\right)$ assigns the data item $\delta_{i}(A)$ to any node $A \in N_{i} \cap \mathcal{K}$.

We then can show that

$$
\left.\mathcal{L}\left(\operatorname{ext}\left(R_{\alpha}\right)\right)\right|_{\mathcal{N}_{\text {ext }}}=\mathcal{L}(\operatorname{ext}(\alpha))^{\omega}
$$

where $\mathcal{N}_{\text {ext }}=\mathcal{N} \cup\left\{A_{\beta}, B_{\beta}: \beta\right.$ is a subexpression of $\left.\alpha\right\}$. As all scheduled-data streams in $\mathcal{L}(\operatorname{ext}(\alpha))^{\omega}$ have the form

$$
\begin{array}{lll}
\left\langle\left\{A_{\alpha}\right\},\left[A_{\alpha} \mapsto 0\right]\right\rangle ; & \Theta_{1} & \left\langle\left\{B_{\alpha}\right\},\left[B_{\alpha} \mapsto 0\right]\right\rangle ; \\
\left\langle\left\{A_{\alpha}\right\},\left[A_{\alpha} \mapsto 0\right]\right\rangle ; & \Theta_{2} ; & \left\langle\left\{B_{\alpha}\right\},\left[B_{\alpha} \mapsto 0\right]\right\rangle ; \\
\left\langle\left\{A_{\alpha}\right\},\left[A_{\alpha} \mapsto 0\right]\right\rangle ; & \Theta_{3} ; & \left\langle\left\{B_{\alpha}\right\},\left[B_{\alpha} \mapsto 0\right]\right\rangle ;
\end{array}
$$

with $\Theta_{i} \in \mathcal{L}(\operatorname{ext}(\alpha)),{ }^{6}$ we may conclude from properties (1)-(4) of the automaton for ext $\left(R_{\alpha}\right)$ and from (*) and (**) that $\mathcal{L}\left(\alpha_{0}\right)$ agrees with $\mathcal{L}\left(R_{\alpha}^{+}\right)$for the final circuit that is obtained from $R_{\alpha_{0}}$ by adding the components "Init" and "Stop" as explained before.

\footnotetext{
${ }^{6}$ One of the $\Theta_{i}$ 's might be infinite in which case the above stream "ends" with $\Theta_{i}$.
} 